\title{
Nucleation and initial propagation of shear cracks
}

\author{
E. Boschi $\left({ }^{*}\right)-$ F. Mulargia $(*)$
}

Received on July 10 th, 1977

SUmmary. - By means of the dislocation theory, an explanation is found for the antientropic process of microcracking-macrocracking transition in the shear fracture initial propagation.

Riassunto. - Per mezzo della teoria delle dislocazioni si trova una spiegazione del processo antientropico di transizione microfrattura-macrofrattura nella propagazione iniziale della frattura di taglin.

The process of propagation of a shear fracture seems to be the basic mechanism responsible of the volcanic phenomena (ANDERSON, and Grew, 1977) ('). From this point of view a volcano is thought of as produced by the progressive opening of a connection between the astenosphere and the Earth's surface, which allows the lavas to ascend.

The physical laws governing the propagation of a fracture ar not well understood. In fact, although this prob'em together with its correlation to the static fatigue and stress corrosion phenomena is of primary interest for any structural project, the solutions proposed in the literature are extremely specialized and valid under very particular conditions. This is due to the fact that these problems have been mainly treated with the purpose of finding empirical laws of easy applicability to special conditions and materials. From the theoretical point of view, very little progress has been made since Griffith's formulation of the brittle fracture theory (GRIFliTH, 1920) $\left({ }^{3}\right)$. The only sensible enhancement has been the inclusion of chemical effects, i. e. stress corrosion phenomena by STUART and ANDERSON (1953) (6). Later, all the efforts have been directed, as we already said, towards

(*) Istituto di Geofisica, Università di Bologna. 
very special lields, mainly because these general theories involve too many unknown parameters to be easily applied to practical computations (Charles and Hillig, 1961; WIEderhorn, 1968; SPEIdel, 1971; SMITH, 1974) $(2,7,5,4)$. However, a correct extrapolation of the shear fracture equations to conditions far outside the laboratory range, such as the Earth's mantle conditions, requires a reliable general model of the propagation of a shear fracture. Until this aim has been accomplished, it will not be possible to find any quantitative solution to the problem of the shear fracture propagation of geophysical interest.

The present work, in which we propose a mechanism for the nucleation and initial propagation of a crack, is intended to be a step towards this goal.

Experimentally we know that the loading of a brittle material under general anisotropic stress conditions leads to the opening of a multitude of micrccracks at stress values much lower than the rupture limits. Increasing the stress to approximately 0.5 the rupture stress, the microcracks coalesce into few macrocracks which beging to propagate. This microcracking-macrocracking transition appears as a general feature of shear fracture dynamics, independently of the material and chemical environment conditions, and obviously is the first phenomenon to analyze in deriving a self-consistent shear fracture theory. We shall subdivide this initial process in two elementary steps, namely:

i) the breaking of some molecular bonds, randomly distributed in the material, due to anisotropies in the stress and in the material itself, with the corresponding opening of microflaws,

ii) the antientropic process of coalescing of these microllaws into a few microcracks which beging to propagate.

We will assume a general material stressed in a general way. The condition for the opening of microllaws at a point $A$ in the material can be then written as

$$
S(A)-T(A)>0
$$

where $S$ is the component of the shear stress parallel to the molecular bond and $T$ is the molecular bond strength. Obviously the set of points $A$ and the $S$-directions, in which the flaws will open, will be determined by local anisotropies in the stress and in the body structures, so that cquation [1] is there maximized. These structure anisotropies depend on very local crystal defects and are impossible to treat theoretically. 
However as it happens in any real situation, there will be, at least locally, some stress symmetry, i. e. an $S$-direction in which the shear stress is maximum. Therefore the instantaneous $S$-axes of a set $\{A\}$ will all tend to orientate along only one direction. We shall look at the cracking on this direction, which we will call $y$. For this purpose we shall treat the microflaw as an edge dislocation distribution $B(v)$. so that the flaw width will be a function of $y$ expressed by

$$
D(y)=\int_{y}^{2 L} B\left(y^{\prime}\right) d y^{\prime}
$$

where $2 L$ is the flaw length. This dislocation distribution produces a shear stress in the medium given by

$$
\sigma(y)=\frac{\mu}{2 \pi} \int_{0}^{2 L} B\left(y^{\prime}\right)\left(y-y^{\prime}\right)^{-1} d y^{\prime}
$$

where $\mu$ is the shear modulus. This stress will tend to inhibit the opening of the flaw and therefore the crack opening condition [1] must be written

$$
S(2 L)-T(2 L)-\sigma(2 L)-\sigma^{\prime}(2 L)>0
$$

where $\sigma$ is the stress tensor near a crack $\gamma$ and generated by it; $\sigma^{\prime}$ is the stress tensor given by the other cracks, which can be written as

$$
\sigma^{\prime}=\int_{\infty}^{0} \sigma(x-\xi)^{-1} d \xi
$$

where $\xi$ is the distance of the other cracks from $\gamma$ measured in the plane orthogonal to $y, x$ being the displacement vector for the crack $\gamma$. Substituting [3] into [5], and using the fact that [3] is monotonically decreasing with $L$ gives that

a) the longer is a crack the easier is its propagation,

b) if a crack is longer than the cthers, for local anisotropies, it propagates absorbing the whole stress energy and inhibiting the growth of other neighboring cracks. 
In conclusion, by the above considerations, we have found a simple physical explanation for the antientropic process of the microcrackingmacrocracking transition in the initial propagation of shear fracture.

\section{ACKNOWLEDGMENTS.}

We wish to thank Professors F. Barberi, P. Gasparini, and G. PUPp1 for their kind support, encouiagment, and suggestions.

This work has been performed in the framework of the activities of the Italian Geodynamics Project, Publ. N. 111.

\section{REFERENCES}

(1) Anderson, O. L., and P. Grew, 1977. - Stress corrosion theory of crack propagation with applications to geophysics. "Rev. Geophys. Space Sci ». 15, p. 77.

(2) Charles, R. I., and W. B. Hillig, 1961. - Kimetics of glass failure of stress corrosion. In «Symposium sur les verres". Florence.

(3) Griffith, A. A., 1920. - The phenomena of rupture and how in solids, "Phil. Trans. Roy. Soc. ». London, Ser. A, 221, p. 163.

(4) Sмiтh, E., 1964. - The structure in the vicinitl of a crak tip. Eng. Fract. Mech., 6, p. 213.

(5) SPEIDEL. M. O.. 1971. - Current understanding of stress corrosion crack growth in aluminium alloys in the theory of stress corrosion cracsing in alloys. Brussels.

(6) Stuart, D. A., and O. L. Anderson, 1953. - Dependence of ultimate strength of glass under constant load on temperature, ambient, atmosphere and time. "J. Am. Cer. Soc.», 36, p. 418.

(7) WIEDERHORN, S. M., 1958. - Moisture assisted crack growlh in ceramics. "Int. 1. Fracture Mech.», 4, p. 171. 